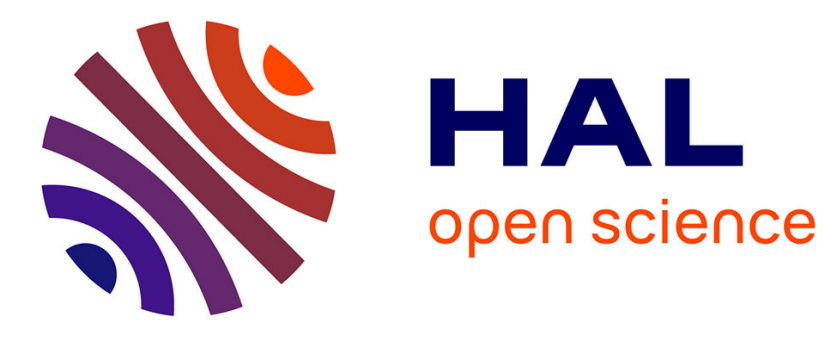

\title{
Improving Energy Conservation Using Bulk Transmission over High-Power Radios in Sensor Networks
}

Cigdem Sengul, Mehedi Bakht, Albert Iii Harris, Tarek Abdelzaher, Robin Kravets

\section{To cite this version:}

Cigdem Sengul, Mehedi Bakht, Albert Iii Harris, Tarek Abdelzaher, Robin Kravets. Improving Energy Conservation Using Bulk Transmission over High-Power Radios in Sensor Networks. International Conference on Distributed Computing Systems, Jun 2008, pekin, China. inria-00333700

\section{HAL Id: inria-00333700 https://hal.inria.fr/inria-00333700}

Submitted on 23 Oct 2008

HAL is a multi-disciplinary open access archive for the deposit and dissemination of scientific research documents, whether they are published or not. The documents may come from teaching and research institutions in France or abroad, or from public or private research centers.
L'archive ouverte pluridisciplinaire HAL, est destinée au dépôt et à la diffusion de documents scientifiques de niveau recherche, publiés ou non, émanant des établissements d'enseignement et de recherche français ou étrangers, des laboratoires publics ou privés. 


\title{
Improving Energy Conservation Using Bulk Transmission over High-Power Radios in Sensor Networks*
}

\author{
Cigdem Sengul \\ ASAP/INRIA \\ cigdem.sengul@inria.fr \\ Mehedi Bakht \\ UIUC \\ mbakht2@uiuc.edu \\ Tarek Abdelzaher and Robin Kravets \\ UIUC \\ $\{$ zaher, rhk\}@cs.uiuc.edu
}

Albert Harris III
CReSIS-KU
afh@cresis.ku.edu

\begin{abstract}
Low power radios, such as the CC2420, have been widely popular with recent sensor platforms. This paper explores the potential for energy savings from adding a highpower, high-bandwidth radio to current sensor platforms. High-bandwidth radios consume more power but significantly reduce the time for transmissions. Consequently, they offer net savings in total communication energy when there is enough data to offset wake-up energy overhead. The analysis on energy characteristics of several IEEE 802.11 radios show that a feasible crossover point exists (in terms of data size) after which energy savings are possible. Based on this analysis, we present a bulk data transmission protocol for dual radio systems. The results of simulations and prototype implementation show significant energy savings at the expense of introducing acceptable delay.
\end{abstract}

\section{Introduction}

Low-power radios (e.g., CC2420 [3]) were introduced to compensate for the excessive idling energy consumption of the high-power radios (e.g., IEEE $802.11 \mathrm{~b} / \mathrm{g}$ radios). Since sensor networks typically experience long idle times but still require very long lifetimes, minimizing idling energy is critical. However, this low idling energy is achieved at the cost of decreased bit rates and increased per-bit energy consumption. While current sensor radios have maximum bit rates in the $100 \mathrm{Kbps}$ range, common IEEE 802.11 radios have minimum bit rates in the Mbps range. Hence, during transmission, the high bandwidth radios have significantly lower per-bit energy consumption.

* This work is funded in part by the National Science Foundation under grants CNS 06-26825, CNS 05-54759, and CNS 06-26342
In an ideal device, the low idling costs would be coupled with the low per-bit transmission energy. To approach this ideal, many power management solutions have been designed for high-power radios. One solution is to sleep cycle the radio, alternating the state of the radio between sleep and idle [6]. However, such sleep cycling cannot reduce the idling energy sufficiently for use in sensor networks. A second solution is to use a low-power radio to wake up the high-power radio [15]. In ad hoc and WLAN networks, where node lifetimes are expected to be on the order of hours or days, the extra cost and complexity of the second radio is the limiting factor in deploying such technology. However, since the expected lifetime for sensor nodes is on the order of weeks or months, the addition of a high-power radio becomes feasible if it can increase node lifetime.

For a dual radio sensor platform, the main challenge is minimizing the amount of time that the high-power radio spends idling. Since the idling energy of the high-power radio is prohibitive, it is imperative to turn it off when not in use. However, transitions from off to on are not free, in terms of time or energy [14]. This transition overhead can be amortized by buffering a minimum amount of data before starting transmission. We call the threshold burst size when it becomes economic to wake up the high-power radio the break-even point. Therefore, sensor nodes must have sufficient memory to buffer enough data to enable energy savings. Until recently, the addition of a high-power radio was not a viable option due to the high memory requirements. However, recent sensor platforms include more powerful processors, memory and bus units (LEAP [11], mPlatform [10]). More importantly, the size of RAM available has significantly increased providing more potential for supporting high-power radios on sensor nodes.

Our contribution is the design of a bulk communication protocol $(B C P)$ that manages the transitions of the highpower radio based on analytic models of the break-even 
point in a dual-radio system. Our analysis is based on the energy characteristics of some off-the-shelf IEEE 802.11 radios and low-power radios used on current sensor nodes, such as Micaz [3]. This analysis shows that for relatively small break-even points, a significant amount of energy can be saved. Results from simulations and our prototype implementation also verify our analytic model. The main side effect of BCP is the buffering delay. Since the delay depends on the time it takes to accumulate data up to the break-even point, different applications will experience different delays based on their data collection rates. As one example, since many environmental monitoring applications measure natural phenomena over long periods of time, a collection delay of even several days is not detrimental, especially if it increases system lifetime. Recent applications, such as EnviroMic [9], where audio is being transmitted through the network, accumulate data much faster making performance almost real-time despite data buffering.

The rest of the paper is organized as follows. In Section 2, we discuss the trade-offs in dual-radio systems and present an analysis of when using a high-power radio is more beneficial compared to using a low-power radio. Our bulk communication protocol, based on this analysis, and its evaluation are presented in Sections 3 and 4, respectively. We conclude and discuss future work in Section 5.

\section{Multi-Radio Communication}

Using multiple radios to reduce the overall energy consumption was initially proposed for WLANs $[17,15,13$, 12]. The main goal is to use low-power radios for less intensive tasks and switch to high-power radios only when necessary. In recent years, new multi-radio sensor platforms have emerged (e.g., Intel Stargate [2], LEAP [11], Intel Mote 2 [1], [8]). The emergence of these platforms is causing a paradigm shift in sensor networks, which were previously considered as low-power, low-data-rate, lowcomplexity networks. However, these new platforms allow more resource-hungry applications, which range from ultralow latency routers [8] to sound collection [4]. Furthermore, we can expect new applications to emerge demanding more resource-rich platforms, completing the innovation cycle.

Dual-radio systems attempt to achieve high-rate communication while maintaining low idling energy use. Since the low-power radio has low idling costs, it can be used for network maintenance. The high-power radio should remain off by default and be turned on only for data communication to achieve better energy-per-bit performance. These transitions from off to on should be managed effectively to guarantee low energy cost. Therefore, in the next section, we analyze the conditions at which dual-radio systems can achieve energy-efficiency.

\subsection{Analysis of a Break-even Point}

The utility of the high-power radio in dual-radio systems is determined by the amount of data communication in the network. Therefore, we define the break-even point as the minimum data size that needs to be accumulated so that a high-power high-rate radio saves energy in comparison to communicating via a low-power low-rate radio. The rest of this section presents a break-even point analysis based on two cases: (a) single-hop, where both radios can reach the same next-hop and (b) multi-hop, where the high-power radio can send multiple hops farther than the low-power radio.

Break-Even Point for the Single-Hop Case To find the break-even point, we calculate the transmission and reception energy expended to transmit and receive data of size $s$. The duration of transmission, and hence reception, is determined by the packet size, $p s^{L}$, the header size, $h s^{L}$, and the rate of the low-power radio, $R_{L}$. The energy consumed by the radios that overhear this transmission is represented as $E_{o}^{L}$. For the analysis, $E_{o}^{L}=0$, since its value depends on the number of neighbors that are awake at the time of the transmission. Although some techniques can be used to reduce overhearing [16], overhearing costs are typically not negligible and hence, we evaluate the case $E_{o}^{L} \neq 0$ in Section 4. Since the low-power radios connect the nodes to form the underlying network, the analysis only includes their transmission, reception and overhearing costs. Their power-management and idling costs are considered to be base costs and not represented in our analysis. Given the transmission and reception powers, $P_{t x}^{L}$ and $P_{r x}^{L}$, the energy cost of using the low-power radio, $E_{L}(s)$ is:

$$
E_{L}(s)=\frac{P_{t x}^{L}+P_{r x}^{L}}{R_{L}} \cdot \sum_{i}^{\left\lceil s / p s^{L}\right\rceil}\left(p s^{L}+h s^{L}\right) \cdot n^{i}+E_{o}^{L} .
$$

Depending on the MAC operation and the channel state, each packet that composes $s$ might be transmitted multiple times. Therefore, the number of transmissions for packet $i$, $n^{i}$, might be greater than 1 . However, for the sake of the analysis, $n^{i}=1$. This assumption is not made in Section 4, where the impact of packet losses is accounted for.

The energy cost of using the high-power radio for data transfer includes the additional costs of waking up the radio at both ends of the communication link. Furthermore, once the node switches on its high-power radio, it spends energy idling in the expectation of the first data packet and between packet receptions. Hence, the energy cost of using the highpower radio for a given rate $R_{H}$ is:

$$
\begin{aligned}
E_{H}\left(s, R_{H}\right) & =E_{\text {wakeup }}^{H}+E_{\text {wakeup }}^{L}+E_{\text {idle }}+E_{o}^{H} \\
& +\frac{P_{t x}^{H}+P_{r x}^{H}}{R_{H}} \cdot \sum_{i}^{\lceil s / p s\rceil}\left(p s^{H}+h s^{H}\right) \cdot n^{i},
\end{aligned}
$$


Table 1. Energy Characteristics $(m W, m J)$.

\begin{tabular}{|l|c|c|c|c|}
\hline Rate & $P_{t x}$ & $P_{r x}$ & $P_{i}$ & $E_{\text {wakeup }}$ \\
\hline Cabletron 2Mbps & 1400 & 1000 & 830 & 1.328 \\
\hline Lucent 2Mbps & 1327.2 & 966.9 & 843.7 & 0.6 \\
Lucent 11Mbps & 1346.1 & 900.6 & 739.4 & 0.6 \\
\hline Mica 40 Kbps & 81 & 30 & 30 & \\
\hline Mica2 38.4 Kbps & 42 & 29 & N/A & \\
\hline Micaz 250 Kbps & 51 & 59.1 & N/A & \\
\hline
\end{tabular}

where $E_{\text {wakeup }}^{H}$ is the energy spent in switching the sender and receiver high-power radios on. The cost of sending messages via low-power radios to wake-up the high-power radio on the receiver side is $E_{\text {wakeup }}^{L} . E_{\text {idle }}$ is the total energy consumed in idling by the two high-power radios. The cost of switching off is negligible, and hence, not represented in (2). $E_{o}^{H}=0$ and $n^{i}=1$ as in (1).

To satisfy $E_{H}(s, R) \leq E_{L}(s)$ :

$$
s \geq \frac{E_{\text {wakeup }}^{H}+E_{\text {wakeup }}^{L}+E_{\text {idle }}}{\frac{P_{t x}^{L}+P_{r x}^{L}}{R_{L}} \cdot\left(1+\frac{h s^{L}}{p s^{L}}\right)-\frac{P_{t x}^{H}+P_{r x}^{H}}{R_{H}} \cdot\left(1+\frac{h s^{H}}{p s^{H}}\right)}
$$

The Equation 3 uses the fact that $n^{i}=1, E_{o}^{L}=0$ and $E_{o}^{H}=0$ for the analysis. The break-even point, $s^{*}$, should satisfy $E_{H}\left(s^{*}, R_{H}\right)=E_{L}\left(s^{*}\right)$.

Break-Even Point for the Multi-hop Case Typically, the transmission range of high-power radios is greater than sensor radios. Therefore, we next explore this case, where it takes multiple hops to reach a certain destination via only low-power radios and the high-power radio can send to the same destination in a single hop for a given rate $R$. Denoting this forward progress $f p^{H}(R)$, the energy consumption for each radio is re-evaluated. The multi-hop energy consumption via low-power and high-power radios, $E_{L}^{m h}(s)$ and $E_{H}^{m h}(s, R)$, respectively, are :

$$
\begin{gathered}
E_{L}^{m h}(s)=f p^{H}(R) \cdot E_{L}(s) \\
E_{H}^{m h}(s, R)=E_{H}(s, R)+\left(f p^{H}(R)-1\right) \cdot E_{\text {wakeup }}^{L}
\end{gathered}
$$

$E_{H P}^{m h}(s, R)$ includes the data transmission energy and the energy used for sending the multi-hop wake-up message. In (5), it is assumed that nodes can send to any destination in a single hop through the high-power radios.

\subsection{Feasibility with Current Cards}

To understand the feasibility of a dual-radio system, we evaluated the break-even points for single-hop and multihop cases for various configurations of three IEEE 802.11 radios (Cabletron, Lucent $(2 \mathrm{Mb} / \mathrm{s})$ and Lucent $(11 \mathrm{Mb} / \mathrm{s})$ ) and three sensor radios (Mica, Mica2, Micaz) (see Table 1

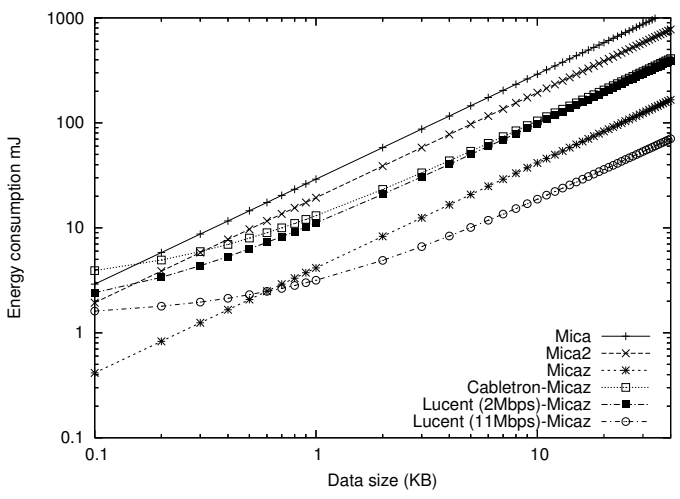

Figure 1. Energy consumption.

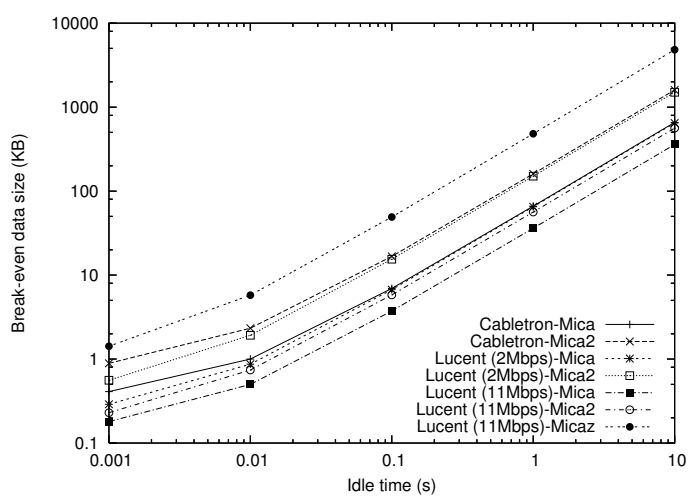

Figure 2. $s^{*}$ as idling time increases.

for energy characteristics). In Fig. 1, energy consumption of single sensor radio platforms, and dual-radio combinations of IEEE 802.11 radios with Micaz are plotted for different data sizes for the single-hop case. The break-even points, $s^{*}$, are the points where the lines for a dual-radio configuration and a sensor radio cross. While $s^{*}$ is typically low (i.e., below $1 K B$ ), there are two cases where using a high-power radio is not feasible. Both Cabletron and Lucent $(2 \mathrm{Mb} / \mathrm{s})$ do not provide any energy savings with Micaz since Micaz has a better energy-per-bit performance than these radios. However, Lucent (11 Mbps) achieves a 50\% energy savings compared to Micaz at around $4 \mathrm{~KB}$.

To represent the energy cost of non-perfect power management for the IEEE 802.11 radio, we next evaluate the effect of idling for the single-hop case (i.e., $E_{i d l e} \neq 0$ in (2)). Obviously, as nodes idle longer (e.g., based on different listen/sleep schedules), $s^{*}$ increases. For instance, when the total idle time is around $1 \mathrm{~s}, s^{*}$ is $66-480 \mathrm{~KB}$ (see Fig. 2). Although this can be easily supported by the new platforms (e.g., Intel Mote 2 [1] has 32 MB RAM), it is still important to minimize the time the IEEE 802.11 radios idle. We 


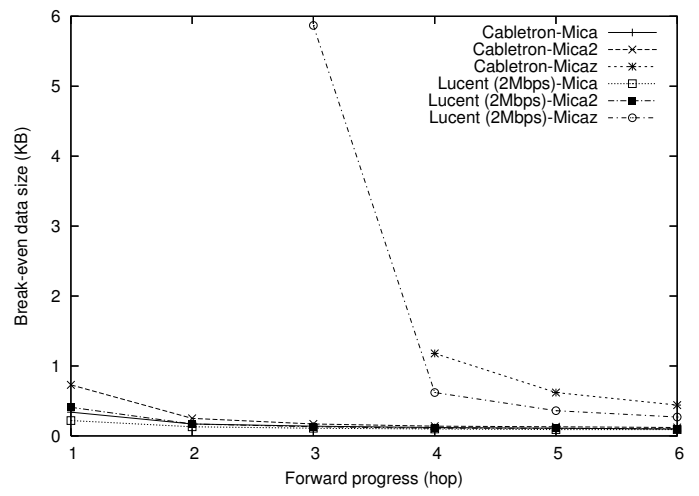

Figure 3. $s^{*}$ as forward progress increases.

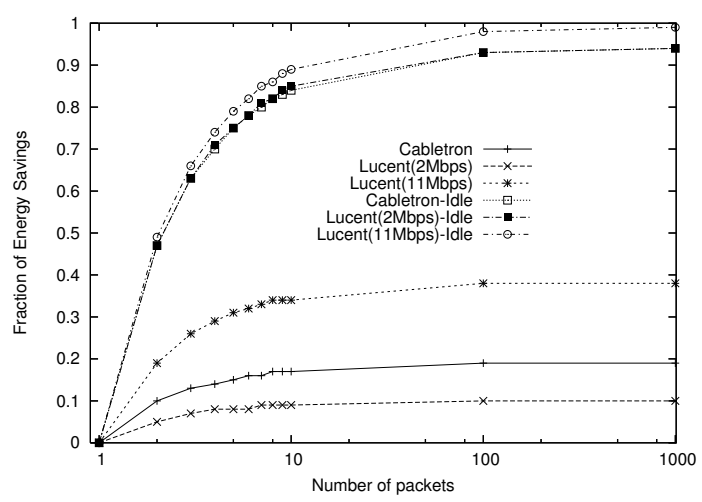

Figure 4. Energy savings with burst size.

discuss some of the design challenges and our solutions to reduce idling in Section 3.

We next show $s^{*}$ for the multi-hop case. The transmission range of IEEE 802.11 radios is approximately $250 \mathrm{~m}$ and the sensor radios is $40 \mathrm{~m}$. The source and the destination are separated by $200 \mathrm{~m}$ in a linear topology. Hence, while the source can reach the destination in one hop with Cabletron and Lucent (2 Mbps), communication through sensor radios require 5 hops. However, as the rate increases, the range that can be supported by the IEEE 802.11 radio decreases. Therefore, we assume Lucent (11 Mbps) has the same range as the sensor radio. As expected, $s^{*}$ for $\mathrm{Ca}$ bletron and Lucent ( $2 \mathrm{Mbps}$ ) radios is lower for the multihop case (i.e., $0.15-0.75 \mathrm{~KB}$ ). Furthermore, Cabletron and Lucent (2 Mbps) configurations become feasible with Micaz when forward progress of these radios is consideredb: the Cabletron - Micaz and the Lucent (2 Mbps) - Micaz combinations become feasible with 4 hops and 3 hops, respectively (see Fig. 3). On the other hand, $s^{*}$ for Lucent (11 Mbps) is the same as in the single hop case.

For both the single-hop and multi-hop case, $s^{*}$ is at most at $1 \mathrm{~KB}$, which corresponds to approximately one packet for IEEE 802.11 radios. Further energy savings can be gained if it is possible to go over $s^{*}$ and send the collected data in larger bursts. However, as the burst size increases, diminishing returns on energy savings are expected. Fig. 4 shows the energy savings from sending $n$ packets in one shot in comparison to waking up $n$ times and sending 1 packet at each awake period. Energy savings increase quickly up to 10 packets (i.e., $10 \mathrm{~KB}$ ) and after this point continue increasing at a slower rate. The energy savings are greater when nodes idle $100 \mathrm{~ms}$ before turning off (labeled as "idle"). Since, in both cases, the majority of savings are obtained when $n=10$, this can be used as the rule of thumb to determine the burst size. We discuss and evaluate other factors that affect the burst size in Sections 3 and 4.

In this section, we have analyzed the feasibility of adding a high-power radio to a sensor node and came to several conclusions. First, there exists a break-even point, $s^{*}$, after which transmitting data using the IEEE 802.11 radio starts saving energy. Second, $s^{*}$ is small enough to fit in the memory in current sensor nodes. Third, higher transmission range of the IEEE 802.11 radio can be leveraged to increase energy savings. Finally, there is a good operating region in terms of burst size, going beyond which does not increase energy savings much. We next describe our bulk communication protocol, which is motivated by this study.

\section{Bulk Communication Protocol (BCP)}

The goal of BCP is to facilitate the accumulation and communication of bulk data to obtain energy savings from the high-power radio. Therefore, a node buffers data until it reaches $\alpha$ times the break-even point $\left(\alpha-s^{*}\right)$, where $\alpha>1$ is a protocol parameter. After this point, the node tries to empty its buffer by initiating a handshake with the receiver. While data packets are buffered, the control messages which are typically small, are sent with the low-power radio to avoid buffering delay. $\mathrm{BCP}$ interacts closely with the routing and the MAC layers. Therefore, next, we explain the interfaces to these layers in detail.

Sender Side: Interface to Routing BCP receives and sends data or control packets to the routing layer. For a packet passed from the routing layer, BCP first needs to identify the type of the packet. While data packets are subject to buffering, control packets are directly passed to the MAC layer of the low-power radio. Data messages for different receivers are buffered separately, so messages for the same next hop can be combined and sent to that next hop.

Sender Side: Interface to MAC layers Once the amount of buffered data for a receiver passes $\alpha-s^{*}$, a wakeup handshake is initiated by sending a wake-up message through the low-power radio. The wake-up message, which contains the burst size, may travel multiple hops to reach the 
receiver (depending on $f p^{H}(R)$, see Section 2.1). When the wake-up message is sent, the sender does not turn its highpower radio on but starts waiting for an acknowledgment (ack) message, which carries the allowed amount of data by the receiver. If this data size is less than $s^{*}$, the sender might give up sending. However, this extension is not evaluated in this work. If the sender times out before receiving an ack, a wake-up message is resent to the receiver. On receiving the wake-up ack, the sender turns its high-power radio on. The allowed amount of data is assembled into packets for the high-power radio and forwarded to the corresponding MAC layer. Therefore, BCP needs to be able to map the low-power and high-power radio addresses for the receiver.

To calculate $s^{*}$, it is necessary to know the energy characteristics of both radios. If these are not known, $\alpha-s^{*}$ can be set, for instance, $10 \mathrm{~K}$ based on our analysis in Section 2.2. Additionally, rate information should be exposed to BCP to calculate $s^{*}$. Currently, the calculation of $s^{*}$ does not include the expected number of retransmissions, since it is hard to predict this number before using the radios. However, due to retransmissions either the low-power or the high-power radio might become more energy-efficient for data transmissions. We leave adapting $s^{*}$ based on retransmissions as future work.

Receiver Side: Interface to MAC layers On reception of a wake-up message, the receiver wakes up its high-power radio and sends back a wake-up ack specifying the amount of data the sender can transmit. If the receiver does not have enough space, the ack message returns a lower burst size. If the receiver's buffer is full, no ack is sent. To avoid waiting for the sender data indefinitely, the receiver times out and turns its high-power radio off if it does not receive any data packets. Data messages are received as an assembly of multiple packets from the MAC layer of the high-power radio and are fragmented into the original packets by $\mathrm{BCP}$. To minimize the time spent in idle, the receiver turns off its high-power radio when it receives the total number of packets advertised or after a timeout.

Receiver Side: Interface to Routing After fragmentation of the messages received via the high-power radio, the original packets are passed to the routing layer. Packets received from the low-power radio that are not wake-up messages are passed directly to the routing layer.

$\mathrm{BCP}$ does not use separate routing layers for different radios. However, the route lookups need the low-power and high-power radio addresses for both the source and the destination to retrieve the correct next-hop. To reduce route discovery overhead of the high-power radios, we advocate using the existing routes over the low-power radios initially and adapting these routes as necessary, similar to route optimizations in [7]. To do such optimizations, the high-power radio on the sender side needs to remain on to hear its packet being forwarded by the intermediate nodes. The last node that forwards the packet is set as the next-hop for the following transmissions. Learning such shortcuts reduces the route discovery overhead for the high-power radios.

\section{Performance Evaluation}

Our performance evaluation extends our feasibility analysis in Section 2.2 to a more comprehensive study via simulations. Furthermore, we demonstrate the feasibility of BCP on actual hardware via a prototype implementation using TinyOS. To understand the impact of BCP on communication performance, we evaluate 3 models: (1) Sensor model, which represents the pure sensor network (2) IEEE 802.11 model, which represents the pure IEEE 802.11 radio network and (3) Dual-radio model, where BCP is used to send wake-up messages via sensor radios and IEEE 802.11 radios are used for data communication. To compare the energy performance, we use the sensor and dual-radio models, since the IEEE 802.11 model has very high energy consumption, even with a power-save mode.

Since our goal is to show the advantage of adding IEEE 802.11 radios to a sensor network, we first use an ideal energy model for the sensor nodes. In other words, only transmit and receive energy is taken into account for the sensor radio, while any cost from idling and overhearing is ignored. On the other hand, any cost from IEEE 802.11 radios (i.e., transmission, reception, overhearing, idling and waking up) is fully charged. Therefore, in terms of energy consumption, the sensor model is shown in the best possible light, while the dual-radio model pays for the cost of the IEEE 802.11 radios fully. Additionally, we evaluate a second sensor model, where nodes are charged for overhearing packet headers (idling costs are still ignored). This second model is essential to understand how moving away from the ideal impacts the energy consumption of the sensor model.

\subsection{Simulation}

We simulate a $200 \times 200 \mathrm{~m}^{2}$ grid network with 36 nodes. Each sender sends its traffic to a sink node. To decouple the routing effects on performance, two separate trees that go over sensor and IEEE 802.11 radios are built. Each simulation is $5000 \mathrm{~s}$. The 802.11 and sensor data packet sizes are $1024 \mathrm{~B}$ and $32 \mathrm{~B}$, respectively. The buffer size is $5000 \times$ 32 B. We evaluate different burst sizes: 10, 100, 500, 1000, $2500 \times 32 \mathrm{~B}$. It must be noted that when 10 sensor packets is chosen as the $\alpha$-breakeven point $\left(\alpha-s^{*}\right), \alpha<1$.

We evaluate the dual-radio performance for the singlehop and multi-hop cases. For the single-hop case, the IEEE 802.11 is Lucent (11 Mbps) and requires the same number of hops as the sensor radio to reach the sink. In the multihop case, the IEEE 802.11 radio is Cabletron and is able to reach the sink in one hop, while the sensor radio requires 


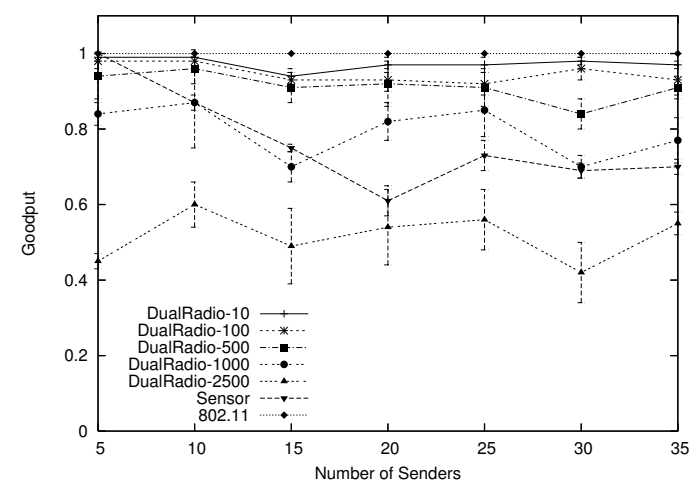

Figure 5. SH: Goodput.

multiple hops. Channel access and retransmissions in the presence of packet losses are handled by full IEEE $802.11 \mathrm{~b}$ MAC layer for the IEEE 802.11 radio. For the sensor radio, we chose a simpler MAC layer that comply with MAC protocols for sensor platforms (e.g., no RTS/CTS). Two radios are assumed to be operating in non-overlapping channels.

The performance metrics are: (1) Goodput, which is the ratio of the number of data bits (excluding overhead) received by the sink to the number of bits transmitted by the senders. (2) Normalized energy (J/bit), the ratio of the total energy consumed by all nodes in the network to the number of bits received by the sink. (3) Delay (s), the difference in time a packet is generated at the sender and received by the sink, including buffering delays. Our results present an average of 20 runs and $95 \%$ confidence intervals.

\subsubsection{Single-Hop (SH) Case}

We first study the case when the IEEE 802.11 radio has no hop advantage but is able to send faster than the sensor radio. Our results show that the goodput of the dual-radio approach is affected by $\alpha-s^{*}$. In Fig. 5, when the burst size is 10,100 and 500 , the dual-radio model performs significantly better than the sensor model and similar to the pure IEEE 802.11 model, where all nodes are active and hence, not limited by the need to wake up and send in bulk. However, as the burst size increases, the goodput degrades because more packets are sent back-to-back multi-hop.

Our results show that, even when the energy costs of the sensor model are ideal, the dual-radio model provides significant savings. The normalized energy consumption of the dual-radio and sensor models is shown in Fig. 6. For the sensor model, both the ideal energy consumption (labeled Sensor-ideal) and the energy consumed in the presence of overhearing (labeled Sensor-header) are plotted. When the burst size is 500 , the normalized energy is $4-5$ times better than the sensor model. More importantly, the dual-radio

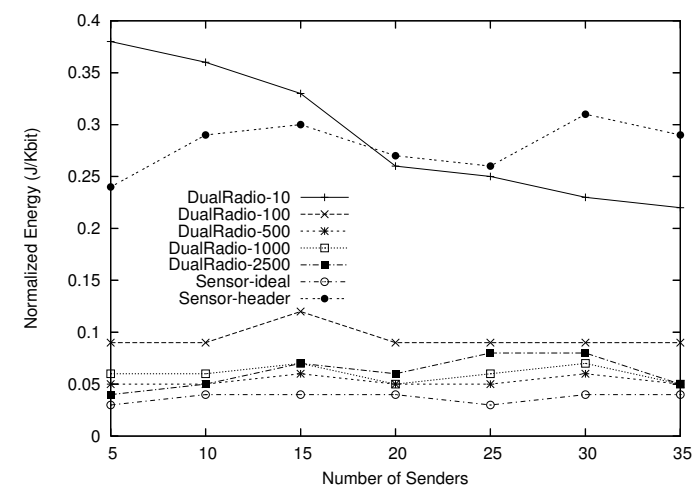

Figure 6. SH: Normalized energy.

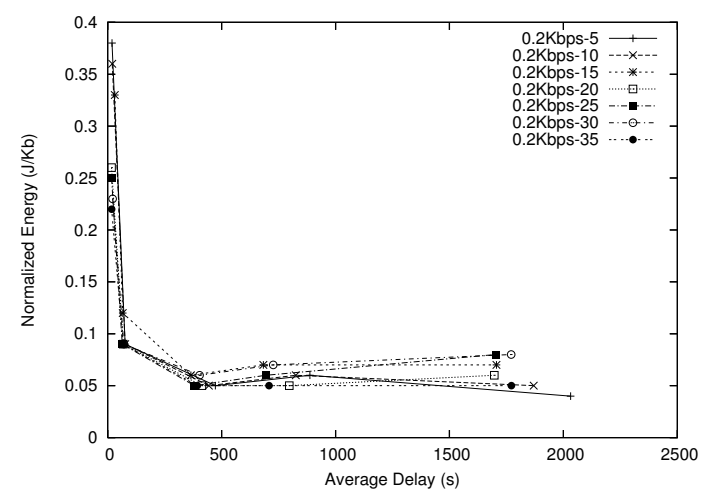

Figure 7. SH: Normalized energy vs. delay.

model approaches the ideal energy consumption of the sensor model. As expected from our analysis, these energy savings are possible only when enough data is buffered and sent in bulk. When the $\alpha-s^{*}$ is 10 (i.e., $320 \mathrm{~B}$ ), and hence less than $1 \mathrm{~KB}$, the dual-radio model does not save energy.

The main side effect of BCP is the extra delay in communication, since data is accumulated up to the $\alpha-s^{*}$ before it is transmitted. In Fig. 7, normalized energy vs. delay for different number of senders is plotted. Each point on a line corresponds to a burst size value. For instance, " $0.2 \mathrm{Kbps}-5$ " refers to the case of 5 senders, and the first point on the line corresponds to the burst size of 10 packets. Fig. 7 shows that the burst size of 500 provides the best energy consumption, whereas the burst size of 100 provides a better energydelay trade-off, and can provide lower delays with a slightly higher energy consumption. Increasing the burst size further increases delay but does not improve energy savings.

\subsubsection{Multi-Hop (MH) Case}

In this section, we study the performance when the IEEE 802.11 radio is able to send to the sink in one hop, while 


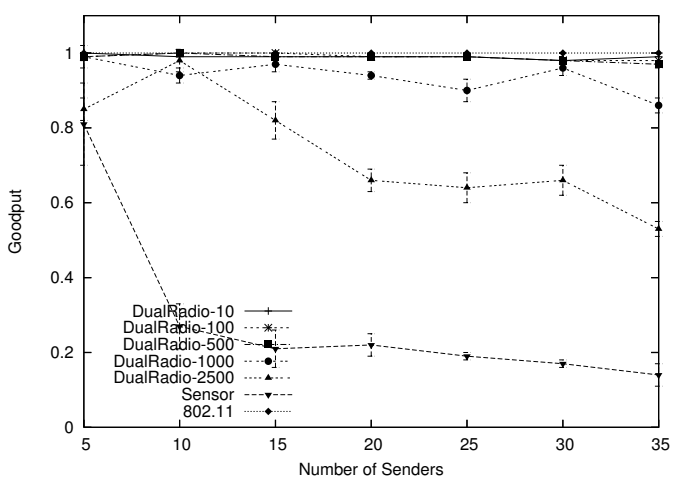

Figure 8. MH: Goodput.

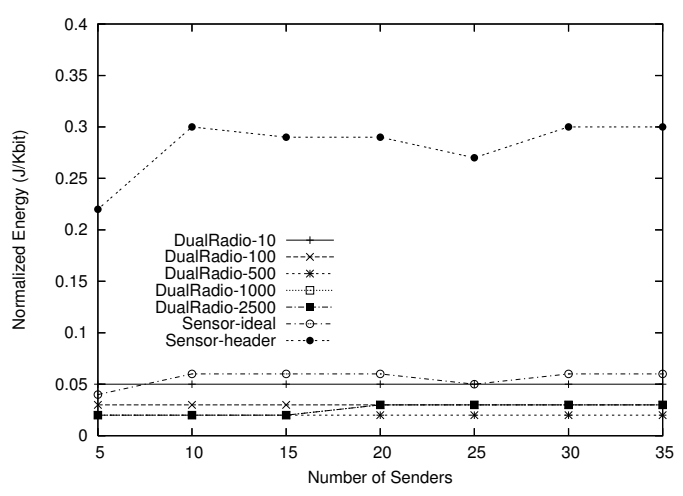

Figure 9. MH: Normalized energy.

the sensor radio requires multiple hops. We have evaluated performance under two different rates: 0.2 and $2 \mathrm{Kbps}$. Due to space limitations, we only present the graphs for $2 \mathrm{Kbps}$ and refer to the results for $0.2 \mathrm{Kbps}$ when necessary.

Fig. 8 shows that the dual-radio model outperforms the sensor model even for the high burst sizes such as 2500 . For the sensor model, the goodput degrades very fast as the number of senders increases due to high contention and packet losses present in multi-hop communication. (This is not the case for $0.2 \mathrm{Kbps}$ and the dual-radio model cannot outperform the sensor model for the burst size of 2500 due to buffering effect.) Most importantly, the dual radio model is able to perform close to or even better than the ideal energy consumption of the sensor model. Even with DualRadio-10 normalized energy improves, mainly due to being able to send in one hop to the sink (see Fig. 9). The lowest energy consumption is achieved when the burst size is $500-1000$ (i.e, 16-32 KB). Increasing the burst size, further increases delays, but does not necessarily save energy (see Fig. 10). (When the traffic rate is $0.2 \mathrm{Kbps}$, the burst size of 10 does not save energy as expected.)

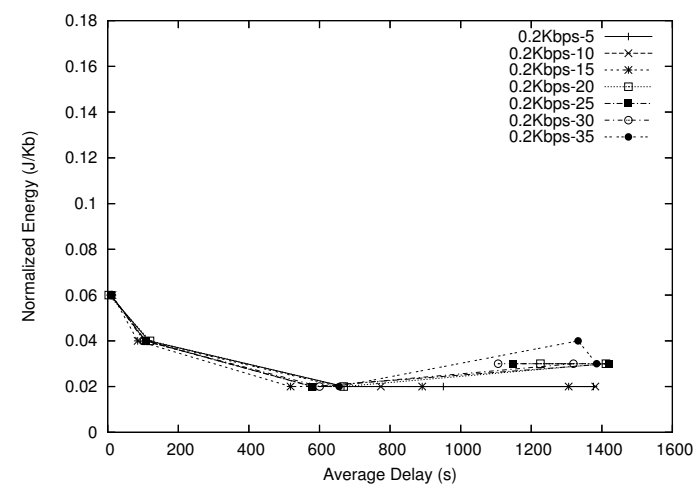

Figure 10. MH: Normalized energy vs. delay.

\subsection{Prototype Experiments}

The initial prototype of $\mathrm{BCP}$ was implemented for the Tmote Sky platform, which uses a single low-power radio (i.e., CC2420). Because the time and energy characteristics of IEEE 802.11 radios have been well studied in literature [5], we chose to emulate the high-power radio. A second MAC interface, which is basically a wrapper around the standard TinyOS MAC interface, was implemented to make the emulation of the IEEE 802.11 radio transparent to BCP. Our main goal is to show how the actual hardware and software environment of sensor nodes, in isolation from other external factors (e.g., interference, bad channel conditions), affect the performance of BCP. To this end, a simple setup of a single sender and a single receiver is used. The results represent the average of 5 runs for increasing $\alpha-s^{*}$, where each run consists of sending 500 messages $(\alpha$ can be $<1$ ). All the events (waking up of the emulated IEEE 802.11 radio, transmission/reception of wakeups, acks, data, etc.) were logged in detail. At the end of the experiments, these logs were used to calculate energy consumption and delay.

Fig. 11 shows the energy consumption per packet for different values of $\alpha-s^{*}$. As expected from the analysis, $s^{*}$ occurs slightly above $1 \mathrm{~KB}$. The energy consumption per packet drops sharply as $\alpha-s^{*}$ increases, but beyond $s^{*}$, the rate of decrease starts diminishing, confirming the analytical results (see Section 2.2). Interestingly, the energy consumption per packet for the dual-radio scheme does not decrease monotonically. This is due to the packet size limitation of the high-power radio. Essentially, a slight increase in $\alpha-s^{*}$ leads to a scenario where the small amount of additional data requires an extra packet to be sent, resulting in an increase in energy consumption on average. Due to the memory constraint of the Tmote Sky, the maximum $\alpha$ $s^{*}$ in our experiments is around $4 \mathrm{~KB}$. However, in sensor platforms equipped with larger memories (e.g., $32 \mathrm{MB}$ RAM in Intel Mote 2 [1]), it is possible to set $\alpha$ larger to 


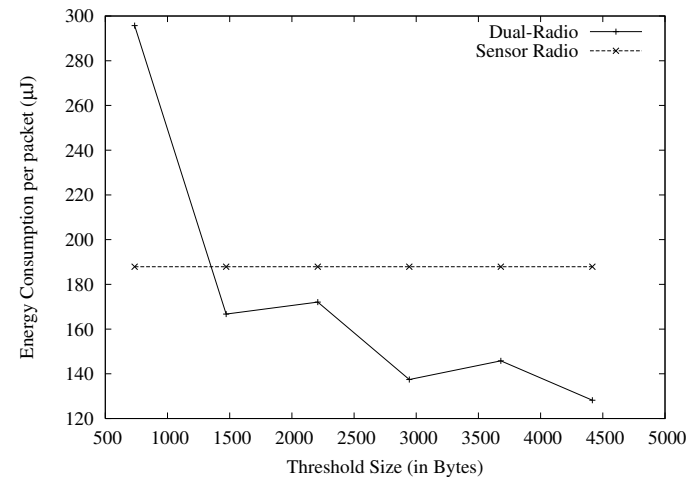

Figure 11. Energy Consumption vs. $\alpha-s^{*}$

obtain higher savings. Finally, we investigate the tradeoffs between delay and energy. Fig. 12 shows that initially, energy consumption can be significantly reduced with increased delay. But beyond a region, increased delay does not improve the energy savings much, validating the similar conclusion derived from the simulation results (Fig. 7).

\section{Conclusions}

Dual-radio platforms are becoming a reality for sensor networks. In this work, we study the feasibility of using a high-power, high-rate radio (e.g., IEEE 802.11 radio) with low-power platforms (e.g., sensor nodes). Our analytical study shows that only a few KBs of data needs to be buffered and sent in bulk to save energy by using the IEEE 802.11 radios. We present a bulk communication protocol (BCP) that handles data buffering, the wake-up handshake through low-power radios and bulk transmissions through the high-power radios. Results from the simulations and implementation of BCP on an emulated dual-radio system confirm that $\mathrm{BCP}$ achieves reduced energy consumption.

In BCP, data messages are always sent by the high-power radio. Based on delay constraints, the low-power radio can also be allowed to send data. However, now, we are faced with the question: is it best to send immediately with the low-power radio or to buffer as much as allowed by the delay constraints and send with the high-power radio? Obviously, we need to know the data patterns to make the right decision. Therefore, we leave using the low-power radio for data communication as future work.

\section{References}

[1] Intel mote 2. http://www. intel.com/research/ downloads/imote_overview.pdf.

[2] Intel stargate. http://platformx. sourceforge. net/index.html.

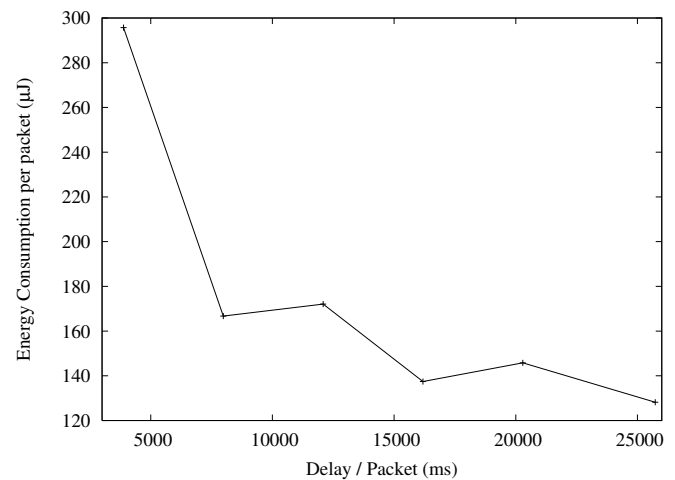

Figure 12. Energy consumption vs. delay
[3] Chipcon wireless technologies presentation, October 2004.

[4] A. M. Ali, T. C. Collier, L. Girod, K. Yao, C. E. Taylor, and D. T. Blumstein. An empirical study of collaborative acoustic source localization. In IPSN, 2007.

[5] L. M. Feeney and M. Nilsson. Investigating the energy consumption of a wireless network interface in an ad hoc networking environment. In IEEE INFOCOM, 2001.

[6] IEEE 802 LAN/MAN Standards Committee. Wireless LAN medium access control (MAC) and physical layer (PHY) specifications. IEEE Standard 802.11, 1999.

[7] D. B. Johnson, D. A. Maltz, and J. Brosch. Ad Hoc Networking, chapter DSR: The Dynamic Source Routing Protocol for Multi-Hop Wireless Ad Hoc Networks, pages 139-172.

[8] M. Kohvakka. Atific helicopter high performance multiradio WSN platform. White paper.

[9] L. Luo, Q. Cao, C. Huang, T. Abdelzaher, J. A. Stankovic, and M. Ward. Enviromic: Towards cooperative storage and retrieval in audio sensor networks. In ICDCS, 2007.

[10] D. Lymberopoulos, N. B. Priyantha, and F. Zhao. mPlatform: A reconfigurable architecture and efficient data sharing mechanism for modular sensor nodes. In IPSN, 2007.

[11] D. McIntire, K. Ho, B. Yip, A. Singh, W. Wu, and W. J. Kaiser. The low power energy aware processing (LEAP) embedded sensor system. In IPSN, 2006.

[12] T. Pering, Y. Agarwal, R. Gupta, and R. Want. CoolSpots: Reducing the power consumption of wireless mobile devices with multiple radio interfaces. In MobiSys, 2006.

[13] J. Redi, S. Kolek, K. Manning, C. Partridge, R. RosalesHain, R. Ramanathan, and I. Castineyra. JAVeLEN- An ultra-low energy ad hoc wireless network. Technical report, BBN, 2006.

[14] C. Sengul, A. Harris, and R. Kravets. Reconsidering power management. In Broadnets, 2007.

[15] E. Shih, P. Bahl, and M. J. Sinclair. Wake on wireless: An even driven energy saving strategy for battery operated devices. In MobiCom, 2002.

[16] S. Singh and C. S. Raghavendra. Pamas: Power aware multiaccess protocol with signalling for ad hoc networks. ACM SIGCOMM CCR, 28:5-26, July 1998.

[17] J. Sorber, N. Banarjee, M. D. Corner, and S. Rollins. Turducken: Hierarchical power management for mobile devices. In MobiSys, 2005. 